\title{
A Microcomputed Tomography-Based Comparison of Root Canal Filling Quality following Different Instrumentation and Obturation Techniques
}

\author{
Anna Kierklo ${ }^{a}$ Zbisław Taborc Małgorzata Pawińskab Małgorzata Jaworska ${ }^{a}$ \\ Departments of a Dentistry Propaedeutics and ${ }^{b}$ Restorative Dentistry and Endodontics, Medical University of \\ Bialystok, Bialystok, and 'Institute of Teleinformatics, Cracow University of Technology, Cracow, Poland
}

\section{Key Words}

Root canal filling · Microcomputed tomography ·

Volumetric analysis

\begin{abstract}
Objective: With a microcomputed tomography (microCT) imaging device, we aimed to quantitatively evaluate root canal fillings after commonly used endodontic procedures and also tested the suitability of microCT for this purpose. Materials and Methods: Eighty single roots were instrumented and obturated with gutta-percha and Tubli-Seal. They were divided into 4 groups of 20. The Hand groups were instrumented with hand files and filled with thermoplastic (Th) compaction and cold lateral (CL) condensation, i.e. Hand-Th and Hand-CL, respectively. The Rot groups, i.e. Rot-Th and Rot- $\mathrm{CL}$, were instrumented with a rotary ProFile system and filled as above. The roots were scanned and 3-dimensional (3D) visualization was obtained. The number, size, percentage of volume and distribution of voids at the filling/dentine interface (i-voids) and voids surrounded by filling material (s-voids) were measured. Results: Canal fillings differed significantly with regard to the size of both types of voids and the average number of $\mathrm{i}$-voids. All canals presented a low
\end{abstract}

volume of voids. The highest percentage $(0.69 \%)$ was found for $\mathrm{i}$-voids in the Hand-CL group, while the lowest volume (0.11\% for s-voids and $0.14 \%$ for $\mathrm{i}$-voids) was in the Hand-Th canals. Apically, in the last $3 \mathrm{~mm}$, i-voids were observed mainly in the Th groups, and s-voids occurred mostly in the coronal part of the canal filling in all cases. Conclusion: MiCroCT was a useful tool for 3D quantitative evaluations of these root canal fillings. None of the root canal instrumentation and filling methods ensured void-free obturation. $\mathrm{CL}$ condensation produced mainly i-voids. With Th compaction, internal s-voids were particularly common, but there were mainly i-voids in the apical part. @ 2014 S. Karger AG, Basel

\section{Introduction}

Due to the multifactorial nature of endodontic treatment, the clinical success of procedures depends on various factors including knowledge of root canal morphology and the whole process from accessing a cavity through instrumentation to the completion of obturation $[1,2]$.

The quality of the root filling is greatly affected by the proper cleaning and shaping of the root canal as well as

\begin{tabular}{|c|c|}
\hline KARGER 125 & $\begin{array}{l}\text { (c) } 2014 \text { S. Karger AG, Basel } \\
1011-7571 / 14 / 0241-0084 \$ 39.50 / 0\end{array}$ \\
\hline $\begin{array}{l}\text { E-Mail karger@karger.com } \\
\text { www.karger.com/mpp }\end{array}$ & $\begin{array}{l}\text { This is an Open Access article licensed under the terms of the } \\
\text { Creative Commons Attribution-NonCommercial 3.0 Un- } \\
\text { ported license (CC BY-NC) (www.karger.com/OA-license), } \\
\text { applicable to the online version of the article only. Distribu- } \\
\text { tion permitted for non-commercial purposes only. }\end{array}$ \\
\hline
\end{tabular}

Assoc. Prof. Dr. Anna Kierklo

Department of Dentistry Propaedeutics

Medical University of Bialystok, ul. J. Waszyngtona 15A

PL-15-274 Bialystok (Poland)

E-Mail ankierklo@op.pl 
the 3-dimensional (3D) hermetic sealing of the root canal system. Obviously, better quality sealing improves the prognosis for the tooth [3]. In order to achieve satisfactory canal preparation, both conventional hand files and nickel-titanium (NiTi) rotary instruments are usually used. The NiTi rotary systems were introduced to reduce the time required for the biomechanical preparation of root canals, especially curved and difficult ones [4-6]. In addition to their great efficiency, these instruments present advantages like flexibility, and they reduce the possibility of apical transportation [7]. Some reports suggest that rotary systems are superior to manual techniques $[6,8]$ and, inversely, some state that neither rotary nor conventional hand files result in a completely prepared root canal surface without tissue remnants $[9,10]$.

Obturation of the root canal must be hermetic to ensure eradication of microorganisms and prevent their regrowth to avoid subsequent periapical diseases [2]. Several studies have shown the high sealing capacity of various filling techniques based on thermoplasticized guttapercha [11-13]. The potential disadvantages of this technique may result in the extrusion of sealer and guttapercha into the periapical tissues. However, CL condensation continues to be a very popular method throughout the world due to its simple execution, low cost and high sealing ability. Disadvantages include the potential lack of homogeneity of the gutta-percha mass $[3,14]$.

The quality of root fillings is often determined by leakage tests and other more precise methods [10, 15-17]. Microcomputed tomography (microCT) can be applied for the analysis of root canal fillings, especially because of their non-homogeneous character and porosity [18-20]. A non-destructive microCT technique provides insight into the details of 3D images of fillings, especially voids and defects, at a level that is impossible to achieve with other methods $[21,22]$. In this context, it would be interesting to assess and compare the quality of canal fillings after commonly used endodontic techniques.

Therefore, the aim of this study was to quantitatively evaluate the root canal obturations with regard to the volume of voids and their distribution in canals filled with CL-condensed and thermoplasticized gutta-percha following manual and rotary instrumentation techniques using non-destructive microCT imaging. Another aim was to test the suitability of microCT for this purpose.

The null hypothesis was that there is no difference between the volume and distribution of voids in the root canals regardless of the studied instrumentation and obturation techniques.

MicroCT of Root Canal Fillings

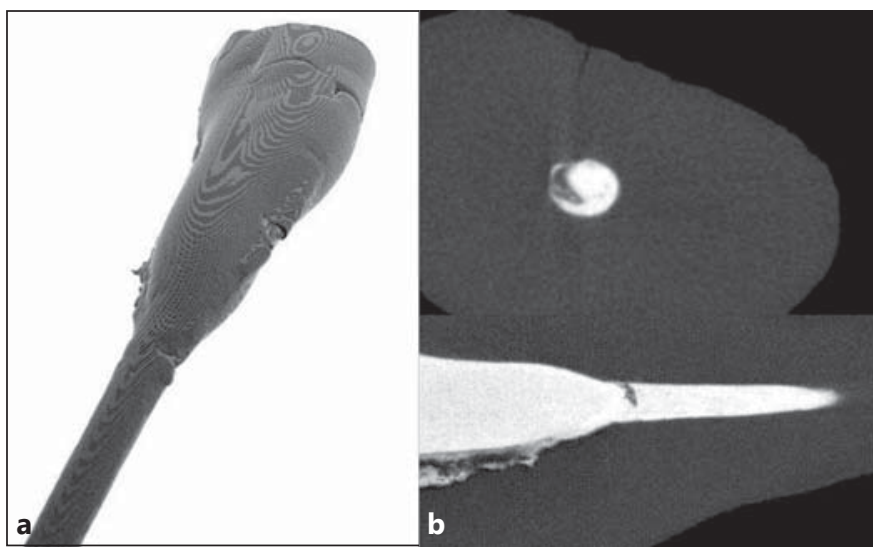

Fig. 1. a Reconstructed 3D image of a tooth. b Perpendicular crosssections of a root and an obturated root canal: background (black), dentine (dark grey) and filling material (white). Voids in the filling material are seen in 2D cross-sections as clusters of dark pixels surrounded by bright pixels.

\section{Materials and Methods}

This project was approved by the University Bioethical Committee prior to the beginning of the experiments. Eighty freshly extracted single-rooted mandibular premolars with a single, straight root canal and fully formed apices were selected. The teeth were free of root caries, cracks and artificial alterations. They were randomly divided into 4 groups of 20 each in order to perform endodontic treatment with two different techniques of preparation and two of canal obturation. They were placed in $5.25 \%$ $\mathrm{NaOCl}$ for $24 \mathrm{~h}$ for surface disinfection and then stored in distilled water until further processing [23]. All specimens were prepared and filled by one operator in order to reduce variability.

\section{Root Canal Preparation}

All teeth were decoronated and the lengths of the roots standardized to the shortest, to achieve a total length of $14 \mathrm{~mm}$. The canals were accessed, and after pulp removal the working length (WL) was established by placing an ISO size-10 K-file (Poldent, Warsaw, Poland) into the canal until visible at the apical foramen and subtracting $1 \mathrm{~mm}$. Orifice enlargement was achieved with Gates Glidden drills (Poldent).

Firstly, 40 root canals (the Hand group) were manually shaped with the step-back technique using size $0.02 / 15-0.02 / 35 \mathrm{~S}$-files (Poldent) to WL, then stepping back $1 \mathrm{~mm}$ with the three subsequent instruments. The canals were irrigated with $2 \mathrm{ml}$ of $2 \%$ $\mathrm{NaOCl}$ solution after the introduction of each instrument size. The next 40 canals (the Rot group) were prepared with a crown-down technique using rotary NiTi ProFile instruments (Dentsply-Maillefer, Ballaigues, Switzerland) prepared according to the manufacturer's recommendations. Instruments were set into permanent rotation with a 4:1 reduction hand-piece (WD-66EM, Buermoos, Austria) powered by a torque-limited electric-motor Endo IT motor (VDW, Munich, Germany). For each file, the individual torque limit and rotational speed programmed in the file library of the Endo IT motor were used. The instrumentation was started with 


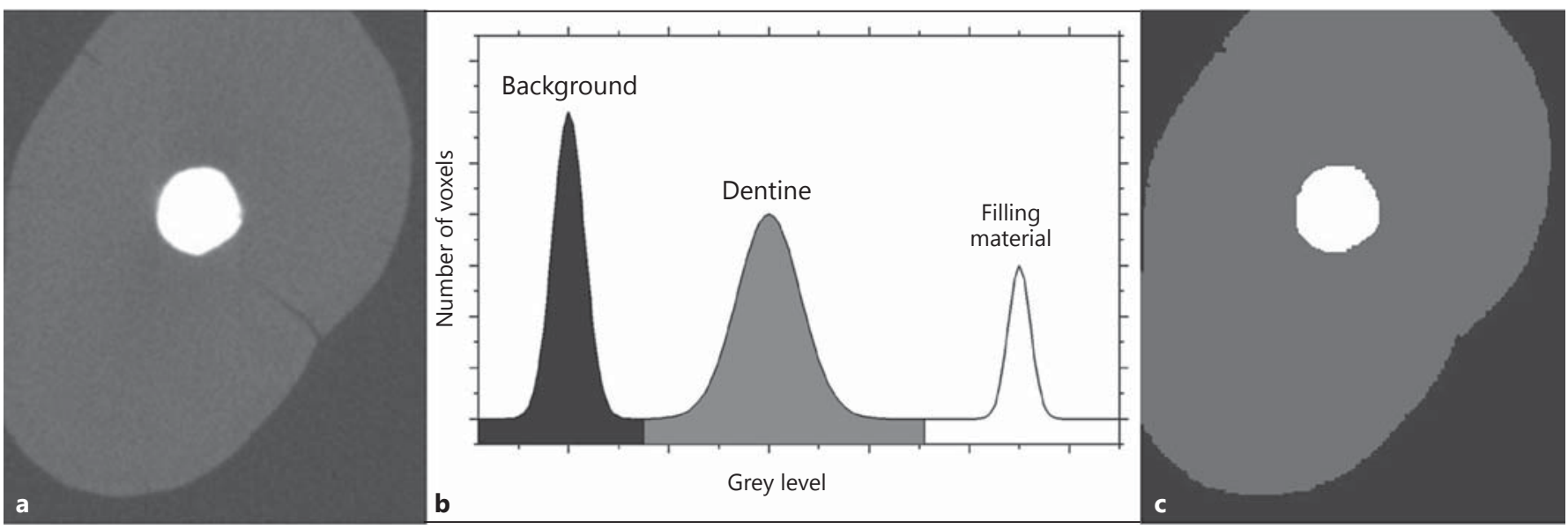

Fig. 2. Stages of the pre-processing of microCT images. a Slice of an original microCT image. b Histogram of microCT data, showing 3 separate modes corresponding to the background, dentine and filling material. c Segmentation of the original microCT data. Voxels with grey levels correspond with background mode (black), dentine mode (dark grey) and filling material (white). the Orifice Shapers $0.07 / 50$ and $0.06 / 40$ to flare the coronal and middle thirds of the root canals. The apical thirds were then prepared in the following sequence: 0.06/30, 0.06/25, 0.04/25, 0.04/30 and $04 / 35$. The final shaping to the WL was achieved when a ProFile instrument size of $0.04 / 35$ in size passed through the WL without force. All canals were irrigated with $2 \mathrm{ml}$ of $2 \% \mathrm{NaOCl}$. After instrumentation, the smear layer was removed by rinsing with $5 \mathrm{ml}$ of $17 \%$ EDTA for $5 \mathrm{~min}$, followed by irrigation with $2 \% \mathrm{NaOCl}$ and saline solution. The canals were then dried with sterile paper points.

\section{Root Canal Filling}

All prepared root canals were obturated with gutta-percha and Tubli-Seal (a zinc oxide-eugenol ZOE-based sealer; Kerr, Salerno, Italy) using two methods: cold lateral (CL) condensation and thermoplastic (Th) compaction. The CL groups, i.e. Hand-CL and Rot$\mathrm{CL}$ were instrumented with hand and rotary files, and the canals were then obturated with the CL condensation technique. Master gutta-percha cones (VDW), 0.02/35 (Hand-CL) or 0.04/35 (RotCL) in size, were fitted into the full WL. They were then coated with a sealer, placed into the root canal and compacted with adjusted finger spreaders to the level of 1-2 mm from the cone tips. After that, accessory cones were inserted until the entire root canal was filled. A heated plugger (Dentsply-Maillefer) was used to cut the coronal surplus, after which the filling was vertically compacted. The Th groups, i.e. Hand-Th and Rot-Th, were obturated by the Th compaction method. The apical part of the canal was sealed to WL with gutta-percha cones (VDW) 0.02/35 (Hand-Th) or $0.04 / 35$ (Rot-Th) in size. Downpacking was performed with a System B plugger (SybronEndo, Orange, Calif., USA), preheating the insert tip to $200^{\circ} \mathrm{C}$. Backfilling was done by injecting heated guttapercha into the canal using an Obtura II unit (Obtura Spartan, Fenton, Mich., USA) according to the manufacturer's instructions.

Complete root canal fillings were confirmed using standard radiographs. Access openings were then sealed with glass ionomer cement (Ketac Cem Radiopaque, 3M ESPE, Seefeld, Germany). The roots were stored at $37^{\circ} \mathrm{C}$ and $100 \%$ humidity for 7 days to allow the sealer to set completely.

\section{MicroCT Evaluation}

An X-tek Benchtop CT160Xi (Nikon Metrology, Tring, UK) microCT scanner was used to scan the teeth, using identical settings for all samples. With the X-ray tube operating at $110 \mathrm{kV}$ and $50 \mu \mathrm{A}$, the samples were scanned with a rotational step of $0.2^{\circ}$ and a rotational angle of $360^{\circ}$, resulting in an isotropic voxel resolution of $10.6 \mu \mathrm{m}$. A $0.25-\mathrm{mm}$ copper filter was used to suppress the beam-hardening artifacts. Raw data were reconstructed with the CT-Pro software using a built-in ring artifact-minimizing algorithm (Nikon Metrology). ImageJ (http://imagej.nih.gov/ij/) was used for 3D volumetric visualization (fig. 1).

Custom-written software was developed to detect voids in the 3D microCT images. Firstly, the images were segmented into three compartments: background, dentine and filling material, based on the analysis of the histogram of grey-level intensities of a microCT image. In all cases, the histogram of grey-level intensities contained three well-separated modes corresponding to the background, dentine and filling material (due to similar radiopacities, we were not able to distinguish between sealer and gutta-percha), so the two segmentation thresholds were set at minima between the modes (fig. 2). After segmentation, voids entirely surrounded by filling material (s-voids) were detected using a hole-filling algorithm [24]. The algorithm starts a search for s-voids by creating a list of all dentine voxels lying at the interface of the background and dentine (i.e. grey voxels which have black voxels in the neighbourhood; see fig. 2c). Then, all the black or dentine neighbours of the list members are subsequently added to the list. The algorithm stops when no new voxel can be added to the list. Clearly, the clusters of grey and black voxels entirely surrounded by white voxels, i.e. the svoids, will never be added to the list and thus monitoring the list content is a direct way to detect s-voids (fig. 3). Finally, concavities present at the interface of the dentine and root canal filling (i-voids) were detected with a watershed from the markers algorithms [25]. Firstly, a Euclidean distance from the filling material (i.e. the white cluster in fig. 3a) was calculated for every dentine voxel, resulting in a distance image (see fig. $3 \mathrm{c}$ for a part of the distance image). The distance function had local maxima at the centres of the interface 

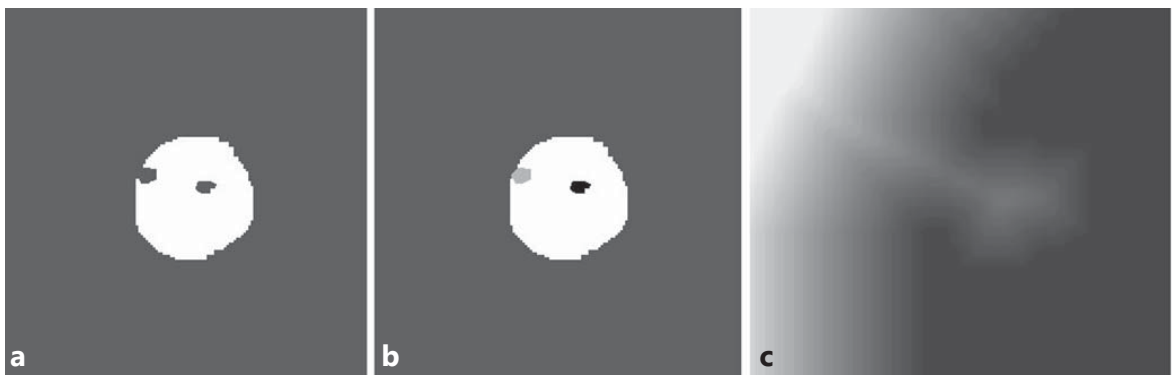

Fig. 3. Stages of the detection of voids. a Segmented microCT slice shows voids within the filling material, i.e. s-voids, or on the interface between dentine and filling material, i.e. i-voids. b S-voids (black) are defined as holes within the filling material and i-voids (light grey) are defined as concavities in the filling material surface. c The i-void part of an Euclidean distance image of $\mathbf{b}$; clusters of white voxels have maxima in the centres of the $\mathrm{i}$-voids which are used to localize the i-voids.

Table 1. Parameters quantitatively characterizing voids in the teeth with root canal fillings

\begin{tabular}{|c|c|c|c|c|}
\hline & Hand-Th group & Rot-Th group & Hand-CL group & Rot-CL group \\
\hline \multicolumn{5}{|l|}{ Internal void (s-void) characteristics } \\
\hline Number of voids ${ }^{a, c}$ & $118 \pm 127(14-546)$ & $31 \pm 24(5-83)$ & $34 \pm 22(5-91)$ & $60 \pm 46(15-184)$ \\
\hline Volume fraction of voids ${ }^{\mathrm{b}}, \%$ & $0.11 \pm 0.12(0.006-0.46)$ & $0.20 \pm 0.30(0.02-1.2)$ & $0.21 \pm 0.18(0.02-0.6)$ & $0.27 \pm 0.28(0.02-1.1)$ \\
\hline \multirow[t]{2}{*}{ Mean size of voids ${ }^{\mathrm{a}-\mathrm{c}}, \mathrm{mm}$} & $0.031 \pm 0.008$ & $0.047 \pm 0.019$ & $0.050 \pm 0.015$ & $0.047 \pm 0.017$ \\
\hline & $(0.018-0.048)$ & $(0.02-0.10)$ & $(0.025-0.80)$ & $(0.026-0.090)$ \\
\hline Mean volume of voids ${ }^{\mathrm{a}-\mathrm{c}}, \mathrm{mm}^{3} \times 10^{4}$ & $0.36 \pm 0.29(0.06-1.1)$ & $1.6 \pm 2.2(0.11-10.0)$ & $1.6 \pm 1.4(0.16-5.2)$ & $1.5 \pm 1.8(0.2-7.4)$ \\
\hline \multicolumn{5}{|l|}{ Parietal void ( $i$-void) characteristics } \\
\hline Number of voids ${ }^{b}$ & $34 \pm 25(8-116)$ & $33 \pm 22(6-83)$ & $56 \pm 35(14-137)$ & $45 \pm 25(7-96)$ \\
\hline Volume fraction of voids ${ }^{\mathrm{a}-\mathrm{c}}, \%$ & $0.14 \pm 0.13(0.005-0.5)$ & $0.55 \pm 0.48(0.05-1.6)$ & $0.69 \pm 0.41(0.05-1.8)$ & $0.52 \pm 0.38(0.12-1.5)$ \\
\hline \multirow[t]{2}{*}{ Mean size of voids ${ }^{a, c}, \mathrm{~mm}$} & $0.053 \pm 0.020$ & $0.069 \pm 0.017$ & $0.066 \pm 0.013$ & $0.066 \pm 0.013$ \\
\hline & $(0.02-0.12)$ & $(0.038-0.097)$ & $(0.037-0.093)$ & $(0.053-0.097)$ \\
\hline Mean volume of voids ${ }^{\mathrm{a}, \mathrm{c}}, \mathrm{mm}^{3} \times 10^{4}$ & $2.3 \pm 3.6(0.09-16.9)$ & $3.8 \pm 2.5(0.5-9.1)$ & $3.1 \pm 1.8(0.5-8.2)$ & $3.2 \pm 2.1(1.5-9.1)$ \\
\hline
\end{tabular}

voids and so markers were automatically placed in these maxima. A watershed from the markers algorithm found concavities corresponding to each marker. Watersheds were calculated for the inverse of the Euclidean distance image and were identified with the basins of attraction of the markers. After detecting the objects of interest, we performed a quantitative analysis of the data.

The absolute volume and coordinates of the centre of mass were calculated individually for each void. The absolute volume of a void is defined as the product of the number of voxels within a void and an absolute volume of a voxel (which is a constant equal to the third power of a voxel size of $10.6 \mu \mathrm{m})$. The centre of mass was calculated in the usual way as an average over coordinates of voxels constituting a void. The $x-y$ plane of the reference frame was parallel to the plane of the microCT slices. The $\mathrm{z}$-axis pointed approximately in the direction of the root axis. The $\mathrm{z}$-coordinate was used to calculate the distance of the voids from the apex. The volume of the root canal filling material (V), s-voids (VS) and i-voids (VI) were also measured, with the volume fraction of the s-voids being equal to the ratio of VS to the sum of V, VS and VI and that of the i-voids being defined analogously. The mean size of the voids was assumed to be equal to the cube root of the absolute volume.

\section{Statistical Analysis}

Means and standard deviations (SDs) were estimated to quantitatively characterize the distribution of the measured parameters. As most of the continuous outcome variables appeared to be extremely skewed, the variables were log-transformed to more normally shaped variables, and then the normality of the distribution of so-transformed variables was assessed with the Shapiro-Wilk test. The differences between the distributions of the same parameters determined for the different obturation/instrumentation methods were tested with a two-way ANOVA with an interaction term included in the model (i.e. an interaction between instrumentation and obturation). Prior to ANOVA testing, the uniformity of variance between groups was assessed with the Levene test. In all cases, a $\mathrm{p}$ value $<0.05$ was considered statistically significant. All statistical computations were performed with Statistica software (StatSoft, Inc., USA). 


\section{Results}

The means, SDs and ranges of all measured parameters are summarized in table 1. All scanned canals demonstrated good filling quality with low volume and volume percentage of voids.

Given the values of the means and SDs, the distribution of the outcome variables appears to be skewed. Indeed, the Shapiro-Wilk test demonstrated that the hypothesis as to the normality of their distribution must be in most cases rejected at the $5 \%$ significance level. For this reason, all variables were log-transformed. In all cases, the hypothesis of the normality of the distribution of the logtransformed variables was not rejected. For all log-transformed outcome variables, the Levene test demonstrated uniformity of variance between all analyzed groups.

With a two-way ANOVA test, it follows that, on average, s-voids were smaller in size than i-voids and the number of s-voids was either higher or lower than the number of i-voids depending on the instrumentation and obturation methods. Statistically significant differences in the average number of s-voids were found between canals instrumented using various methods ( $\mathrm{p}$ value $=0.049$ ). The number of s-voids was higher in the groups instrumented using the hand technique. The average number of s-voids in the Hand-Th group was significantly higher than in all the other groups. Statistically significant $(p=0.0047)$ differences in the average number of $\mathrm{i}$-voids were found between canals obturated with both techniques, to the disadvantage of CL condensation as opposed to Th compaction. Both types of voids were significantly smaller in size ( $p=0.0165$ for $\mathrm{i}$-voids and $\mathrm{p}=0.0463$ for $\mathrm{s}$-voids) for the manually prepared canals (Hand group) compared to those prepared with ProFile instrumentation (Rot group). In contrast, the largest empty spaces were noticed among the i-voids. Significant differences between canals obturated with the cold and warm methods were also found with regard to the size of the s-voids $(p=0.0024)$. All canals presented low percentages of voids in total volume ranging from 0.11 to $0.69 \%$. Statistically significant $(\mathrm{p}=$ 0.0035 ) differences in the percentage of s-voids of the total volume of root canal filling (volume fraction of s-voids) were found for the Th versus the CL canals. The highest percentage of volume $(0.69 \%)$ for the i-voids was found in the Hand-CL group, while the lowest volume $(0.11 \%$ for the s-voids and $0.14 \%$ for i-voids) was in the Hand-Th canals. The canal fillings of the Hand-Th group differed significantly from the other groups. In particular, the number of s-voids was higher $(\mathrm{p}=0.0001)$ and the size of the $s$-voids, the size of the i-voids and the volume fraction of

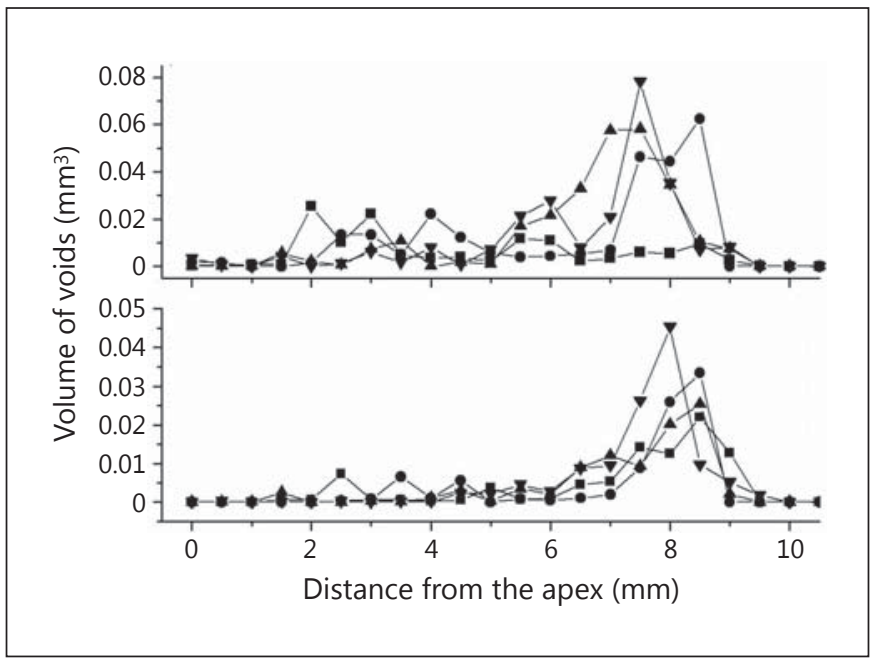

Fig. 4. Longitudinal distribution of the volume of voids along the root canal filling. The volume of voids localized at a specified distance from the apex is plotted against this distance for the 4 groups of analyzed specimens, i.e. Hand-Th ( $\boldsymbol{\square})$, Rot-Th (•), Hand-CL ( $\Delta)$ and Rot-CL ( $\boldsymbol{\nabla})$ for the parietal i-voids (top) and internal svoids (bottom).

the $\mathrm{i}$-voids were smaller than in the other groups $(\mathrm{p}=$ $0.0028, p=0.0246$, and $\mathrm{p}=0.0001$, respectively).

The average distribution of voids in the volume of specimens is shown in figure 4 . It follows from the figure that, in the last 3-4 $\mathrm{mm}$ from the apex, voids were present mainly in the roots filled with the Th compaction method; i-voids occurred on the entire surface of contact with the walls of the root canal obturation and s-voids mostly in the coronal part of the canal obturation. For all the examined methods, the largest total volume of both s-voids and i-voids was observed in the coronal part of the root.

\section{Discussion}

In our investigation, a 3D microCT analysis was successfully applied to effectively visualize and assess detailed information on voids created within the filling and between the filling and the canal walls. A comprehensive multiparametric assessment was provided of two types of voids within the root canal obturations after the most commonly used endodontic techniques. Relatively numerous samples were analyzed in comparison to other microCT-based studies $[13,15,18,26]$.

Our results demonstrated ex vivo good sealing ability of techniques frequently used in daily endodontics, al- 
though none of the instrumentation/obturation combinations evaluated resulted in void-free root canal fillings. This outcome is consistent with the observations of other authors $[18,26,27]$. Nevertheless, the null hypothesis, which stated that all applied treatment methods are equivalent, was rejected. These differences were found with respect to both the preparation procedures and the canal obturation techniques. In particular, we found that the groups of obturated canals differed significantly in terms of the number of voids and the size and percentage of voids in the total volume in favour of hand instrumentation and the Th obturation techniques. After CL condensation, more parietal voids with a high percentage of volume were noticed. I-voids may be in contact with potentially infected canal walls and may promote microleakage and, ultimately, the clinical failure of root canal treatment. Keçeci et al. [27], in a stereomicroscopic examination, showed no significant relationship between the preparation and obturation techniques in terms of the distribution of filling material or voids. However, sectioning does not provide volume information, and thus better precision can be expected from a microCT-based assessment of voids.

Several authors have claimed that NiTi instruments have markedly improved the quality of root canal shaping compared with their stainless steel counterparts, because they produce a well-tapered root canal form suitable for obturation [8]. However, they still produce microdefects and irregularities on the dentine surface which may remain unfilled $[14,18,22]$. The popular system ProFile instrument is designed to U-file, with cutting edges that help keep the instrument in the centre of the canal [7]. On the other hand, the traditional hand biomechanical instrumentation technique resulted in less untouched surface areas than in the case of rotary files; this may be related to the use of hand files in a circumferential filing motion and an anticurvature direction of filing [6].

In this study, we demonstrated that in canals which were manually instrumented, the voids of the canal filling were observed more frequently, but that after using the ProFile system, parietal voids were significantly larger with both filling methods. This result can be partially explained by the findings of the aforementioned studies. Indeed, less untouched surface areas of the canal walls after hand instrumentation compared with the rotary technique should lead to a smaller average volume of parietal voids.

Several studies have confirmed that warmed guttapercha is more adaptable to the irregularities in the canal walls, enabling better sealing than with traditional CL condensation $[15,28]$. However, it has been suggested that using the Th method might have a negative effect on the apical seal, at least initially, when the gutta-percha cools [17]. It has been found that the combination of sealer contraction and air entrapment during Th compaction promotes apical void formation [29]. Our findings are consistent with these. We observed that i-voids were particularly common in the apical third of the canal with Th compaction.

Our results indicate that Th compaction of gutta-percha resulted in both types of voids, with the majority of the voids being surrounded by filling (s-voids) when compared with the parietal i-voids, which, in turn, were less numerous than in the CL condensation of gutta-percha. Moreover, these voids were more compact than those formed after CL condensation. It may be due to their small size and volume that they were not noticeable on conventional and digital radiography or even on spiral tomography [30]. According to Zakizadeh et al. [19], the numerous small s-voids in the coronal part of the filling decrease obturation homogeneity which may promote microleakage from the oral environment. The cause of this pericoronal filling porosity might be the inaccurate and too-weak condensation of the injected warm guttapercha, resulting in void entrapment in the widest part of the canal. In contrast, Lea et al. [31] reported that Th compaction produced an obturation with greater density than conventional CL condensation in vitro. On the other hand, Keçeci et al. [27] reported that CL condensation had a similar incidence of voids in comparison with the System B technique.

With regard to CL condensation, the final filling is composed of several gutta-percha cones tightly pressed together but still remaining separate, so canal filling is less homogeneous than after application of the heated guttapercha technique [15]. According to Peng et al. [3], its poorer adaptation to the canal wall can be the result of poor canal preparation, inadequate pressure during condensation or a mismatch between the gutta-percha cones and the prepared root canal dentine. This could explain the fact that, in our study, a lot of unfilled spaces were identified mainly as the voids formed between the material and the canal wall. I-voids occupied the largest percentage of the total canal filling volume, but s-voids were also spacious. With CL condensation, only the apical third was found to be homogenous, and microCT revealed the presence of voids in the middle and coronal thirds. Gandolfi et al. [18] also observed that coronal thirds contain voids, whereas apical thirds were relatively free from voids. 
MicroCT analysis was used to study Th compaction or CL condensation of root canal fillings using different sealers: AHPlus, AH26, MTA, Sealapex and Tubli-Seal $[18,20,21,26]$. Hammad et al. [26] demonstrated that the application of Tubli-Seal induced fewer voids and gaps than GuttaFlow and resin-based sealers. So far, no sealer with ideal properties exists. ZOE-based sealers have been used for many years with clinical success in spite of their viscosity, porosity, lack of chemical adhesion to the dentine and solubility in tissue fluids [29]. Some studies have shown that volumetric expansion of gutta-percha occurs when in contact with eugenol, and that ZOE-based sealers exhibit low shrinkage [32]. In addition, ZOE-based sealers, in contrast to AHPlus (epoxide-based ones), are characterized by a radiographic density and radiopacity similar to gutta-percha. The sealers are more radiopaque than dentine, affording differentiation from the canal wall in X-ray examinations, with a low risk of artifacts [33].

Our results show that microCT is a tool providing an insight into the quality of root canal filling. Indeed, using microCT, quantitative assessment of canal filling volume can be performed without destruction of the teeth. Although a high correlation between microCT images and histological cross-sections of root canal fillings has been reported [20, 21], Zaslansky et al. [20] argue that some dose of caution is necessary before analyzing the results of studies of interfaces in root canal fillings based on microCT, light microscopy and electron microscopy.

As with other methods, microCT has several limitations which are to be considered before drawing conclusions from the data. Firstly, in this study, the minimal size of voids which could be detected using mictoCT was directly related to the voxel size of the microCT images; voids that occupied only a few voxels could not be distinguished from the background due to the partial volume effect. The sensitivity of microCT to the detection of voids is also limited by other factors, with beam hardening probably the most significant $[20,21]$. Beam hardening artifacts are especially intrusive for their marked differences in contrast to the materials present in the field of view, with a seriously deteriorated image quality disabling the reliable detection of voids. In order to suppress these artifacts, we chose filling materials that would minimize the difference between the radiopacities of gutta-percha, sealer and dentine. Note that, for polychromatic X-ray beams, the problem of beam hardening cannot be fully addressed even if dedicated filters are used. Results obtained by microCT can be underestimated as a consequence of the dehydration and possible dimensional changes that accompany the prolonged scanning and reconstruction time [20]. To minimize these adverse effects, we kept the roots in a water environment except for during scanning.

\section{Conclusion}

MicroCT was a sensitive and useful tool for the 3D quantitative evaluation of these root canal fillings. None of the root canal instrumentation and filling methods evaluated in this study ensured void-free obturation in the whole canal. CL condensation produced voids mainly between the canal wall and the filling. With Th compaction, the internal voids were particularly common, except for in the apical third of the canal where there were mostly parietal voids. Despite this, the results suggest that all the studied techniques of instrumentation and obturation of the root canal are acceptable and effective in clinical practice.

\section{Acknowledgement}

This research was supported by the Medical University of Bialystok, Poland (grant Nos. 113-91814 L and 133-91907 L).

\section{References}

1 Ureyen Kaya B, Keçeci AD, Guldas HE, et al: A retrospective radiographic study of coronal-periapical status and root canal filling quality in a selected adult Turkish population. Med Princ Pract 2013;22:334-339.

2 Kalender A, Orhan K, Aksoy U, et al: Influence of the quality of endodontic treatment and coronal restorations on the prevalence of apical periodontitis in a Turkish Cypriot population. Med Princ Pract 2013;22:555-560.
Peng L, Ye L, Tan H, et al: Outcome of root canal obturation by warm gutta-percha versus cold lateral condensation: a meta-analysis. J Endod 2007;33:106-109.

4 Guelzow A, Stamm O, Martus P, et al: Comparative study of six rotary nickel-titanium systems and hand instrumentation for root canal preparation. Int Endod J 2005;38:743752 .
Loizides AL, Kakavetsos VD, Tzanetakis GN, et al: A comparative study of the effects of two nickel-titanium preparation techniques on root canal geometry assessed by microcomputed tomography. J Endod 2007;33:1455-1459.

6 Yin X, Cheung GS, Zhang C, et al: Microcomputed tomographic comparison of nickel-titanium rotary versus traditional instruments in C-shaped root canal system. J Endod 2010;36:708-712. 
7 Iqbal MK, Firic S, Tulcan, et al: Comparison of apical transportation between ProFile and ProTaper NiTi rotary instruments. Int Endod J 2004;37:359-364.

8 Peters O, Schonenberger K, Laib A: Effects of four NiTi preparation techniques on root canal geometry assessed by microcomputed tomography. Int Endod J 2001;34:221-230.

-9 Peters O: Current challenges and concepts in the preparation of root canal systems: a review. J Endod 2004;30:559-567.

10 Zmener O, Pameijer CH, Banegas G: Effectiveness in cleaning oval-shaped root canals using anatomic endodontic technology, ProFile and manual instrumentation: a scanning electron microscopic study. Int Endod J 2005;38:356-363.

11 Schilder H: Filling root canals in three dimensions. Dent Clin North Am 1967;11:723-744.

12 Vizgirda PJ, Liewehr FR, Patton WR, et al: A comparison of laterally condensed guttapercha, thermoplasticized gutta-percha, and mineral trioxide aggregate as root canal filling materials. J Endod 2004;30:103-106.

$\checkmark 13$ Somma F, Cretella G, Carotenuto M, et al: Quality of thermoplasticized and single point root fillings assessed by micro-computed tomography. Int Endod J 2011;44:362-369.

-14 Shemesh H, Wesselink PR, Wu MK: Incidence of dentinal defects after root canal filling procedures. Int Endod J 2010;43:9951000.

-15 Marciano MA, Ordinola-Zapata R, Cunha TVR, et al: Analysis of four gutta-percha techniques used to fill mesial root canals of mandibular molars. Int Endod J 2011;44:321-329.

-16 Wu MK, Kast'akova A, Wesselink PR: Quality of cold and warm gutta-percha fillings in oval canals in mandibular premolars. Int Endod J 2001;34:485-491.
$17 \mathrm{Xu}$ Q, Ling J, Cheung GS, et al: A quantitative evaluation of sealing ability of 4 obturation techniques by using a glucose leakage test. Oral Surg Oral Med Oral Pathol Oral Radiol Endod 2007;104:e109-e113.

18 Gandolfi MG, Parrilli AP, Fini M, et al: 3D micro-CT analysis of the interface voids associated with Thermafil root fillings used with AH Plus or a flowable MTA sealer. Int Endod J 2012;46:253-263.

19 Zakizadeh P, Marshall SJ, Hoover CI, et al: A novel approach in assessment of coronal leakage of intraorifice barriers: a saliva leakage and micro-computed tomographic evaluation. J Endod 2008;35:871-875.

20 Zaslansky P, Fratzl P, Rack A, et al: Identification of root filling interfaces by microscopy and tomography methods. Int Endod J 2011; 44:395-401.

21 Jung M, Lommel D, Klimek J: The imaging of root canal obturation using micro-CT. Int Endod J 2005;38:617-626.

22 Metzger Z, Zary R, Cohen R, et al: The quality of root canal preparation and root canal obturation in canals treated with rotary versus selfadjusting files: a three-dimensional microcomputed tomographic study. J Endod 2010; 36:1569-1573.

23 Somma F, Leoni D, Plotino G, et al: Root canal morphology of the mesiobuccal root of maxillary first molars: a micro-computed tomographic analysis. Int Endod J 2009;42:165174.

24 Soille P: Morphological Image Analysis, Springer, Berlin, 1999.
25 Vincent L, Soille P: Watersheds in digital spaces: an efficient algorithm based on immersion simulations. IEEE Trans Pattern Anal Mach Intell 1991;13:583-598.

26 Hammad M, Qualtrough A, Silikas N: Evaluation of root canal obturation: a three-dimensional in vitro study. J Endod 2009;35:541544.

27 Keçeci AD, Celik Unal G, Sen BH: Comparison of cold lateral compaction and continuous wave of obturation techniques following manual or rotary instrumentation. Int Endod J 2005;38:381-388.

28 De Deus G, Gurgel-Filho ED, Magalhães KM, et al: A laboratory analysis of gutta-perchafilled area obtained using Thermafil, System $\mathrm{B}$ and lateral condensation. Int Endod J 2006; 39:378-383.

29 Mokeem-Saleh A, Hammad M, Silikas N, et al: A laboratory evaluation of the physical and mechanical properties of selected root canal sealers. Int Endod J 2010;43:882-888.

30 Anbu R, Nandini S, Velmurugan N: Volumetric analysis of root fillings using spiral computed tomography: an in vitro study. Int Endod J 2010;43:64-68.

31 Lea CS, Apicella MJ, Mines P, et al: Comparison of the obturation density of cold lateral compaction versus warm vertical compaction using the continuous wave of condensation technique. J Endod 2005;31:37-39.

32 Chandrasekhar V, Morishetty PK, Metla SL, et al: Expansion of gutta-percha in contact with various concentrations of zinc oxide-eugenol sealer: a three-dimensional volumetric study. J Endod 2011;37:697-700.

-33 Tanomaru-Filho M, Jorge EG, Tanomaru JM, et al: Evaluation of the radiopacity of calcium hydroxide- and glass-ionomer-based root canal sealers. Int Endod J 2008;41:50-53. 